\title{
ANTROPOLOGÍA \\ Iconología de las coyas en la Crónica de Guaman Poma de Ayala, siglo XVII
}

investigaciones sociales

Vol.23 N. ^43, pp.15-23 [2020]|UNMSM/IIHS. Lima, Perú

DOI: http://dx.doi.org/10.15381/is.v23i43.18483

Recibido: 29/04/2020
Aprobado: 04/05/2020
Publicado: $25 / 08 / 2020$

\author{
Patricia Estefany Palomino Osorio \\ Universidad César Vallejo, \\ <patriciapalomino.osorio97@gmail.com>
}

\author{
Miguel Antonio Cornejo Guerrero \\ Universidad Nacional Mayor de San Marcos \\ <mcornejog@unmsm.edu.pe>
}

\begin{abstract}
RESUMEN
Planteamiento del problema. ¿Qué relación existe entre el análisis iconológico de las coyas a través de la cultura material en la crónica de Guaman Poma de Ayala? Objetivo. Definir la relación entre el análisis iconológico de las coyas a través de la cultura material de Guaman Poma de Ayala. Antecedentes. Alvez (2016); Baulenas (2015); Días (2015); Falconi (2017); Parani (2019); Sanchez (2014); Ulloa (2015). Marco teórico. Serán usadas tres teorías para esta investigación; análisis iconológico (Panofsky 1957), las coyas (Guaman Poma 1615; Alvez 2016), y cultura material (Carreton 2017; Igareda 2011). Metodología. El tipo de investigación es aplicada, de diseño no experimental y enfoque cuantitativo. La población fue censal y comprende las 12 láminas de las coyas en la obra del cronista. En el que se usó la lista de cotejo de 36 ítems. La confiabilidad se midió con el método del KR 20 a través del software Excel y SPSS. Resultados. Las coyas llevaban una indumentaria y un estilo de vida muy diferente respecto al resto de la población, teniendo ropa ornamental e incluso "sirvientes", como el tupu, la ystalla, acsu, lliclla, entre otras. Conclusiones. Como conclusión general se debe mencionar que la relación entre el análisis iconológico y la cultura material, se relacionan de manera que permite identificar el origen y vida de las coyas. Recomendaciones. Se sugiere, poder incorporar temas nuevos en cuanto a la elaboración de tesis, para de esta manera motivar a futuros investigadores.
\end{abstract}

Palabras clave: Iconología; coyas; cultura material; análisis iconológico; diseño gráfico.

\section{Coyas iconology in Guaman Poma de Ayala chronicle during the 17th century}

\begin{abstract}
Statement of the problem. What is the relationship between the iconological analysis of the coyas through material culture in the Guaman Poma de Ayala chronicle? Objective. Define the relationship between the iconological analysis of the coyas through the material culture of Guaman Poma de Ayala. Background. Alvez (2016); Baulenas (2015); Días (2015); Falconi (2017); Parani (2019); Sanchez (2014); Ulloa (2015). Theoretical framework. Three theories will be used for this investigation; iconological analysis (Panofsky 1957), the coyas (Guaman Poma 1615; Alvez 2016), and material culture (Carreton 2017; Igareda 2011). Methodology. The type of research is applied, of non-experimental design and a quantitative approach. The population was census and includes the 12 plates of the coyas in the work of the chronicler. In which the 36 item checklist was used. Reliability was measured with the KR 20 method through Excel and SPSS software. Results. The coyas wore clothing and a very different lifestyle compared to the rest of the population, having ornamental clothing and even servants, such as the tupu, ystalla, acsu, lliclla, among others. Conclusions. As a general conclusion, it should be mentioned that the relationship between iconological analysis and material culture are related in a way that allows the origin and life of the coyas to be identified. Recommendations. It is suggested to be able to incorporate new topics regarding the thesis elaboration, in order to motivate future researchers.
\end{abstract}

KeYwords: Iconology; Coyas; Material culture; Iconological analysis; Design. 


\section{Introducción}

\subsection{Planteamiento del problema}

$\mathbf{L}$ as soberanas coyas, esposas de los inkas reinantes, se diferenciaban del resto de las mujeres inkas, incluso en sus vestimentas tradicionales, tenían el privilegio de estar cerca al Inka, compartían su poder y se encargaban del bienestar del pueblo, representaban la comunidad, eran respetadas y se cumplían todas sus órdenes, solían estar acompañadas por sus "sirvientes". En esta investigación se profundiza el rol que desempeña una coya en el Tawantinsuyu y el papel de su vestimenta, que las hacia resaltar por su singularidad de diseños en su lliclla, acsu y otras prendas. Por consiguiente, nuestro objetivo principal es definir el vínculo entre el análisis iconológico de las coyas a través de la cultura material en la crónica de Guaman Poma, y como específicos, definir la relación entre escena, características, política y sociedad en relación a la cultura material en la crónica de Guaman Poma.

La crónica habla acerca de las doce coyas, las que presentan distintos elementos que las diferencian y asemejan, entre ellas y ante el resto de mujeres del Tawantinsuyu. Es por ello, que queremos saber ¿Qué relación existe entre el análisis iconológico de las coyas a través de la cultura material en la crónica de Guaman Poma de Ayala? También, qué relación tienen las dimensiones establecidas como escena, características, política y sociedad esto en conjunto con la segunda variable la cual es cultura material.

\subsection{Antecedentes}

Alvez (2016) en su obra titulada La mujer Inka según el cronista Guaman Poma de Ayala (siglos XVI al XVII), nos narra sobre el cronista y su aporte a la historia, para centrarse en el estudio de las mujeres Inkas o también llamadas coyas. Las compara con los ideales europeos de una mujer. Barraza (2012), en su investigación Acllas y personajes emplumados en la iconografía alfarera Inka: Una aproximación a la ritualidad prehispánica andina, intentó resaltar la importancia que posee el subestilo alfarero Cuzco Policromo Figurado, como nueva fuente informativa sobre las prácticas rituales y algunas de las concepciones religiosas más trascendentales de la sociedad inka.
A partir del análisis de dos motivos reproducidos en este tipo de cerámica, los personajes femeninos con toca cefálica (PFTC) y personajes masculinos emplumados (PME), fueron identificados. Los diseños antropomorfos, ofrecen una vía directa y segura para el reconocimiento de las identidades y funciones sociales, cumplidas en tiempos prehispánicos, por los grupos representados pictóricamente.

Baulenas (2015), su investigación Señora de todas las tierras: Mama Ocllo y el papel de la coya en el Tahuantinsuyo es un estudio de la coya y la importancia que tenía para el pueblo.

Dias (2016), en su tesis titulada Muerte y escatología: transcendencia medieval a través de la imagen. Un análisis historiográfico, iconográfico e iconológico sobre la muerte en la sociedad medieval y en la tradición pictórica del norte de Europa en los siglos XV y XVI, tiene como objetivo, identificar la imagen y su cambio en las creencias de un sistema, también, sobre la tradición funeraria y todo lo relacionado a la muerte.

Sánchez (2014), en su tesis titulada Iconología simbólica en los bordados populares toledanos, tiene como objetivo lograr un análisis de las piezas digitales e indagar en el arte de la confección más relevante del bordado toledano del siglo XVI-XX.

Ulloa (2015), en su tesis titulada Cultura material, patrimonio arqueológico y diáspora africana en la República Dominicana. Un enfoque crítico-epistemológico, establece el objetivo de investigar la cultura material sobre zonas del Caribe, viendo así identidades que los representan.

\section{Marco teórico}

Usamos tres teorías para esta investigación: análisis iconológico (Panofsky 1957), las coyas (Guaman Poma 1615; Alvez 2016), y cultura material (Carreton 2017; Igareda 2011). Según Panofsky (1957), en el nivel iconológico se investiga todo lo relacionado al contexto cultural que desempeńa una obra, además se observa de una forma más compleja datos que no pueden ser vistos a primera instancia, para descubrir los significados de cada elemento en un determinado tiempo (p. 31). Según Guaman Poma (1993 [1615]) los Inkas se casaban con su hermana o prima, para conservar y no arriesgar la mascaypacha en la panaqa gobernante. También, según su cosmología, prevale- 
cía la pureza de la sangre de los hijos del sol, es así que la mujer coya era reconocida como un personaje muy importante del Tawantinsuyu (p. 118). Alvez (2016), menciona que la función de la mujer coya estaba establecida como hija, madre y esposa, se encargaban del aspecto económico, ya que ellas administraban su hogar, incluso la función pública (párr. 7). Según García (2016), la tecnología ayuda a ordenar y elaborar las cosas más comunes como simples, y se basa en el conocimiento científico, que tiene pasos como indagar, diseñar piezas de diferentes materiales y darle diferentes usos, además del mantenimiento que tiene esta tecnología (p.12).

\section{Metodología}

El diseño de la investigación es no experimental, de enfoque cuantitativo, de tipo aplicada, el alcance es explicativo. Se tuvo una población censal, debido a que se tomó en cuenta las doce láminas de las coyas, para ello se usó la lista de cotejo como instrumento y para poder analizar los datos, la fórmula del KR20 en el programa Excel y SPSS.

\section{Resultados}

\subsection{En cuanto a los resultados del análisis descriptivo}

El cronista emplea planos en sus láminas para diferenciar a las coyas del resto de los elementos. Sin embargo, solo algunas están en primer plano, para destacar al personaje icónico, y en otras no, porque para Guaman Poma, no solo las coyas podrían informar, sino también, los "sirvientes". La mayoría de las coyas no están presentes en el interior de una hacienda, ya que para ellas la mejor manera de ayudar a su pueblo es estar en contacto con ellos, mientras que otras coyas se dedicaban más en su hacienda para recibir a los invitados del inka. Se observa la presencia de haciendas, con ventanas y alfombras en su interior. Se observa a las coyas normalmente al aire libre, solo una minoría se encontraban en una hacienda. A la séptima coya se le ve acompañada de un mono, era amiga de los animales. Con la séptima y doceava coya, aparecen papagayos y guacamayos.
Se aprecia una importante relación entre el personaje principal y la talla. Pues el cronista representa a las coyas con más talla, en relación con el resto de personajes en la escena. El cronista representa a la novena y décima coya, con estatura baja. La mitad de las coyas usaban el cabello tapado con la Iñaca. La mayoría de las coyas usaba el cabello rizado, incluso se aprecia la costumbre en otras mujeres. La quinta coya posee el cabello desarreglado, pues sufrió un ataque al corazón. La mitad de las coyas son delgadas y de rostro refinado, la otra mitad lo presenta ovalado. A la mayor parte le gustan las flores, solo a dos de las coyas, Cusi Chimbo Mama Micay y Rau Ocllo, mandaban hacer grandes fiestas para las personas principales, las cuales le traían hermosos presentes. A la sexta coya le gustaba tocar el tambor y las fiestas, la danza y el canto. La mayoría de coyas se interesaban por el pueblo y ayudaban a los ciudadanos. Por ejemplo, a la primera coya, Mama Ocllo, se le atribuían milagros y curaba a los enfermos mediante hechicería. Solo se ven a la primera, sexta y doceava coya, en el importante papel de dirigir y gobernar al pueblo inka. La cuarta, la sexta y la décima coya, aparecen con lacayos viejos en las escenas representadas en las láminas, cabe resaltar que ni el propio inka aparece con estos personajes. La mayoría de las coyas eran representadas ostentando jerarquía social, aparecen con criadas que las acicalaban, una coya tenía criadas enanas.

Todas las coyas llevan lliclla, un manto representado en distintas formas y diseños y tocapus, diseños de formas geométricas, que se colocaban en la indumentaria, como la lliclla y otros, considerados una forma de escritura en la cultura inka. Todas aparecen con maceteros y flores que decoraban sus haciendas.

La mayoría de las coyas presenta el tupu, elemento distintivo de su estatus, de distintos tipos morfológicos; llevaban la Iñaca, un cubrecabeza; aparecen representadas con alfombras en sus haciendas y usando uxota, es decir, sandalias o calzado.

Solo algunas coyas presentaban quitasol, un accesorio que era una especie de sombrilla que cubría a la coya mientras que una de sus "sirvientas" lo sostenía; presentaban ystalla, una especie de cartera o bolso, en que llevaban hojas de coca; llevaban una pequeña vasija que servían de herencia para sus hijos. Llevaban espejo y peine. 


\subsection{En cuanto a los resultados del análisis inferencial}

\subsubsection{Correlación entre análisis iconológico y cultura} material

Como el nivel de significancia es mayor a 0 , la correlación de las variables es positiva moderada, aceptando así la hipótesis de investigacion y rechazando la nula. El análisis iconológico permite el estudio de la simbología, en cuanto a toda presentación de artes visuales, comportamiento de la persona, la forma de vestir o la apariencia física. Tiene relación con la cultura material, para saber a qué grupo pertenecían estos objetos de estudio y transmitir la caracterización de las coyas, demostrando la significancia de la vestimenta y el significado particular de estas doce coyas, así como su rol como esposa y de aspectos políticos, sociales y religiosos.

\subsubsection{Correlación entre escena y cultura material}

Como el nivel de significancia es mayor a 0 , la correlación es positiva moderada, aceptando así la hipótesis de investigación y rechazando la nula. La escena ayuda a entender el ámbito y contexto cultural de los personajes y objetos asociados. Precisamente es la recreación expuesta en una determinada composición, de cómo los personajes, principales y secundarios, aparecen en distintos planos, jerarquizando la escena. En primer plano estarían las coyas y en el plano secundario, los "sirvientes". La cultura material cobra significado en distintos niveles gracias a la escena.

\subsubsection{Correlación entre caracteristicas y cultura material}

Como el nivel de significancia es mayor a 0 , la correlación es positiva alta, aceptando así la hipótesis de investigación y rechazando la nula. La correlación de las características y la cultura material, aumenta la posibilidad en la recopilación de información específica de la vida de las doce coyas, como sus cualidades, personalidad y su aspecto físico.

\subsubsection{Correlación entre politica y cultura material}

Como el nivel de significancia es mayor a 0 , la correlación de las variables es positiva muy baja, aceptando así la hipótesis de investigación y rechazando la nula. En la cultura inka, se sabe que el poder máximo lo tenían los hombres, pero para que esto ocurra, era requisito estar casados, solo así podrían iniciar su gobierno y tomar decisiones en beneficio del pueblo. Las coyas aportaron en diferentes aspectos políticos y de gobierno, ellas se encargaban de las necesidades del pueblo y enseñaban el cuidado de la familia.

\subsubsection{Correlación entre sociedad y cultura material}

Como el nivel de significancia es mayor a 0 , la correlación de las variables es positiva moderada, aceptando así la hipótesis de investigación y rechazando la nula. Las coyas tuvieron un importante rol social, demostrado por la jerarquía alcanzada en la panaqa y representada por su vestimenta y ornamentos jerárquicos, propios de su cultura material inka. La presencia de sus criados en diferentes escenas, demuestra su posición social. Estas señoras organizaban grandes fiestas o rituales, donde participaba el pueblo y consolidaban su posición social.

\section{Discusión}

Para las investigaciones realizadas y mencionadas en los antecedentes, el análisis iconológico y la cultura material ayudó a recolectar información de las láminas de las coyas en la simbología de cada elemento que los acompaña a las coyas, es decir el estudio y la identificación del rol de la mujer inka como parte influyente de una sociedad importante como lo fue la cultura Inka.

Alvez (2016), en su investigación La mujer Inka según el cronista Guaman Poma de Ayala (siglos XVI al $X V I I)$, aporta a la historia una valiosa información de las coyas, por ejemplo, en qué consistía su papel al ser la esposa del Inka, desde el punto de vista social, político y religioso. Es así que esta investigación está de acuerdo con el autor mencionado, ya que se encontró similitud con lo planteado en cuanto el rol de la mujer Inka en las doce láminas de las coyas, puesto que ellas eran encargadas de estar en contacto con su pueblo, y veían de qué manera podían ayudar y enseñar las labores domésticas a las demás mujeres, actividades que se realizaban en ese tiempo para que prevalezcan las buenas costumbres, como era el tejido para las mujeres una herencia que se trasmite en generaciones. Además, las coyas eran las únicas que tenían el privilegio de hablar con sus criadas y lacayos a comparación del Inka. 

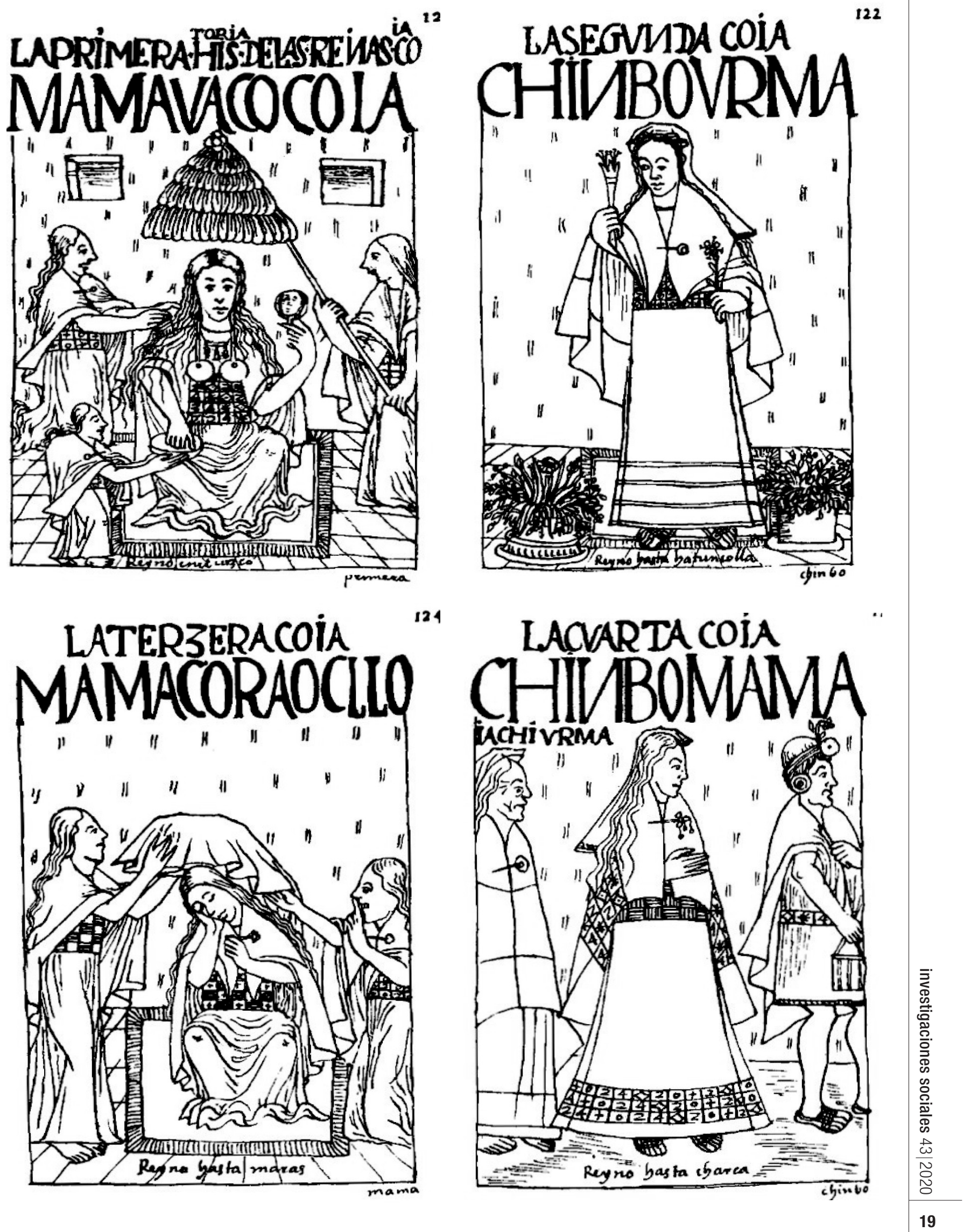
$12 \mathbf{z}$ CHIIBOMAMA

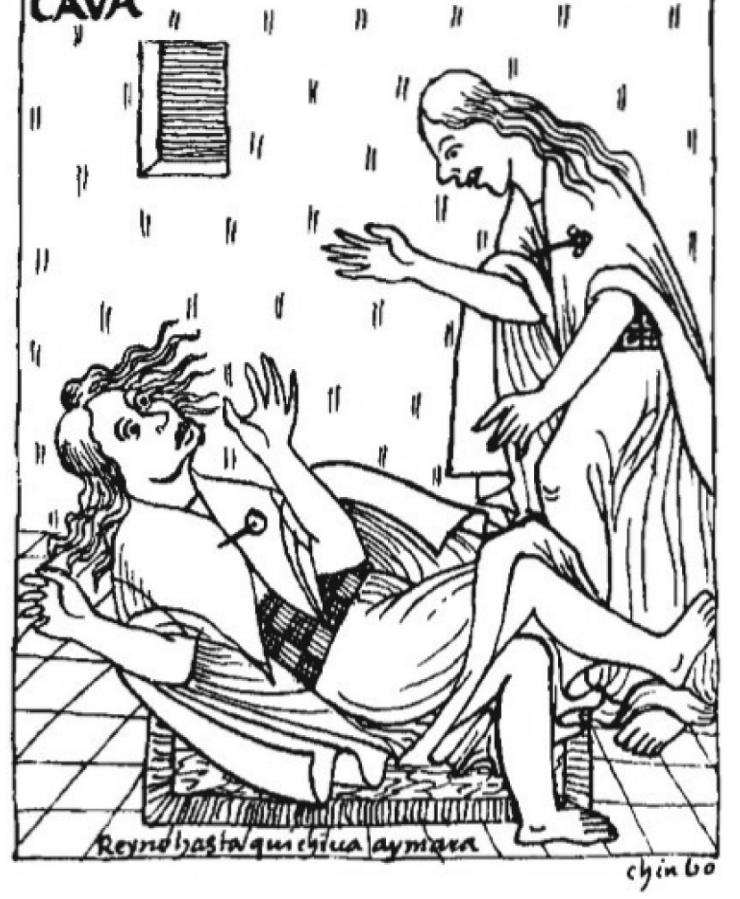

LAQVIUTACOIA

LASES TA COIA CVCICHEBBOMAMA micai "

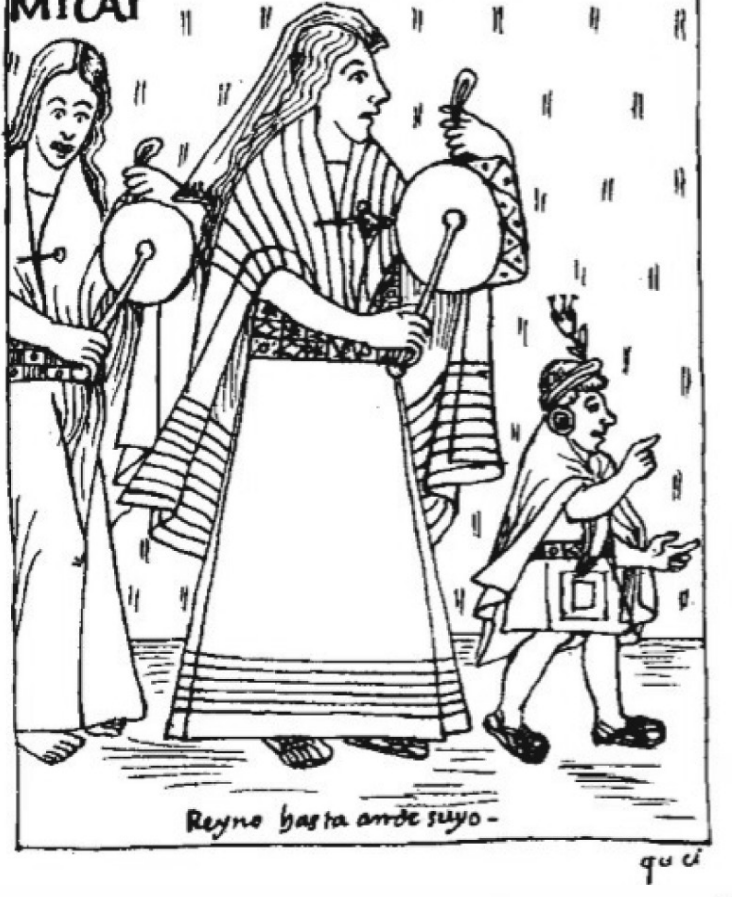

132

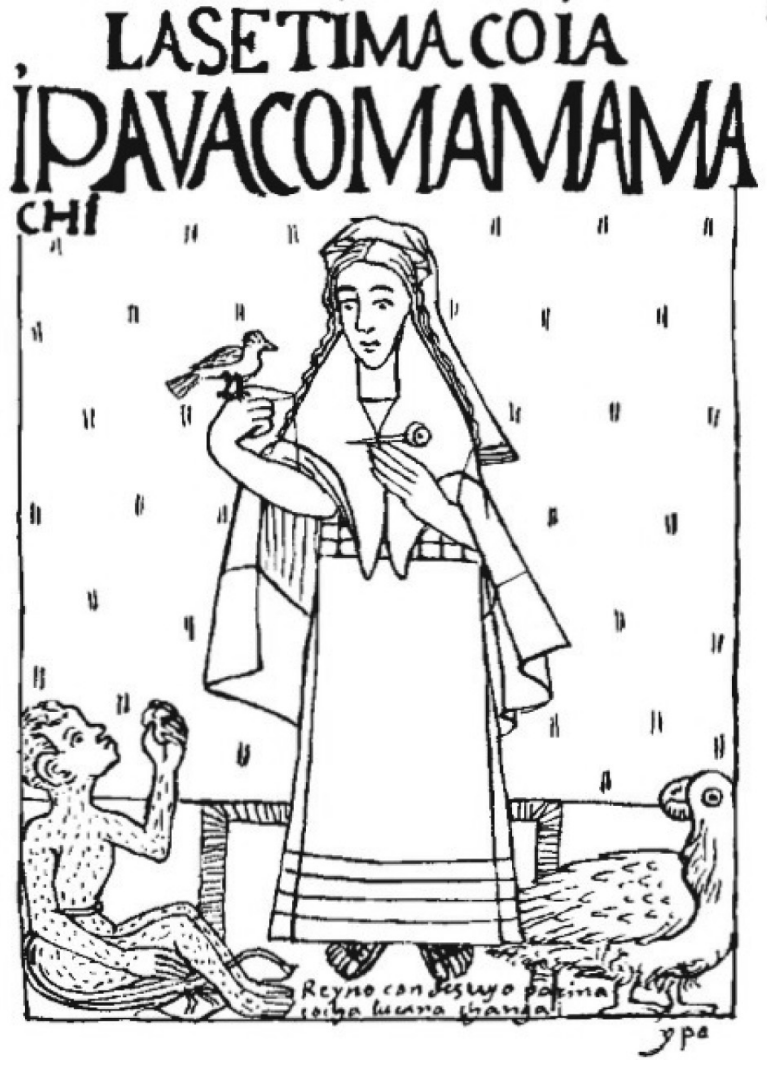



laotava coía MAMAMИTOCA An" " " " " " " 

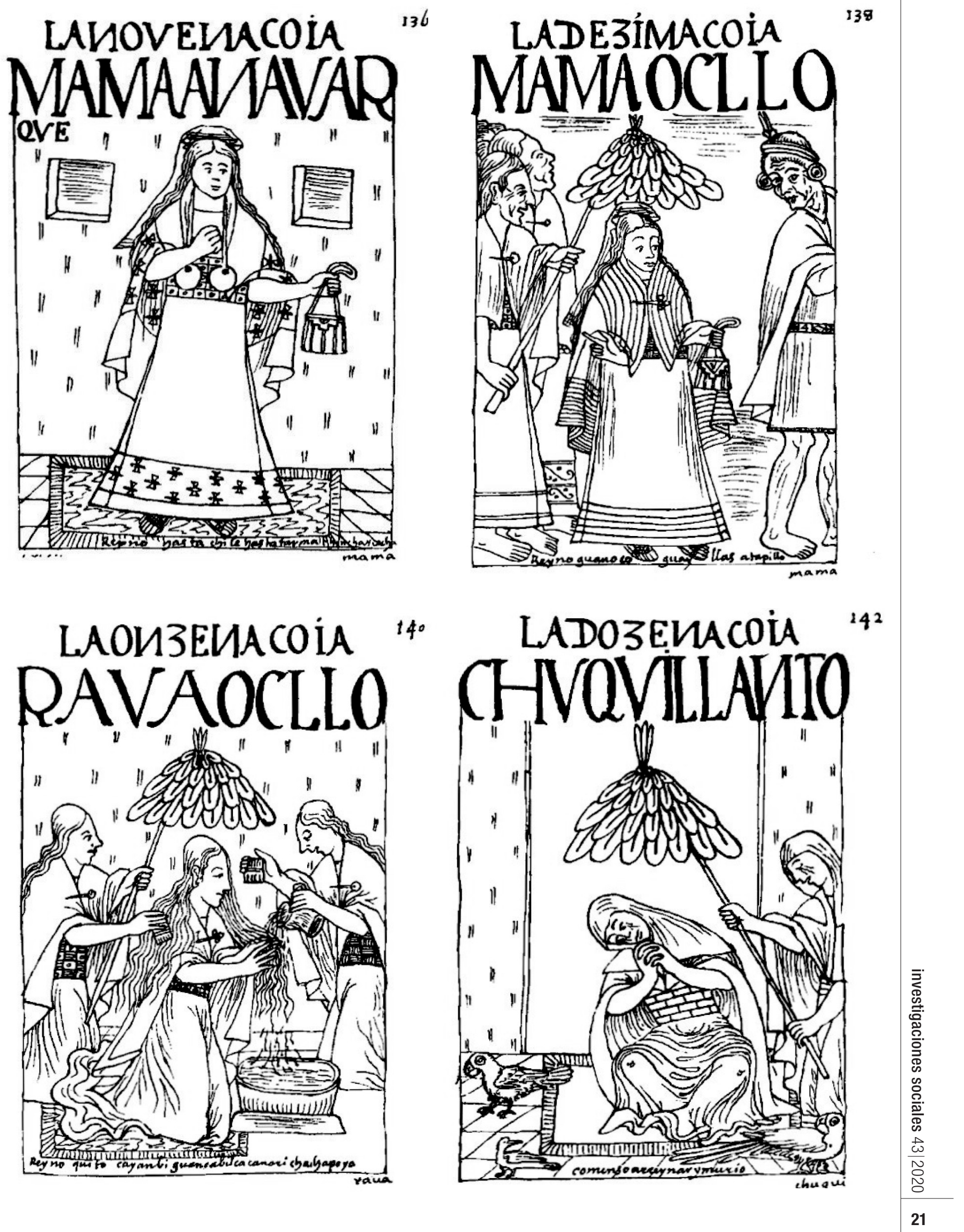
Para Baulenas, en su investigación Señora de todas las tierras: Mama Ocllo y el papel de la coya en el Tawantinsuyu, su materia de estudio es la coya como personaje principal y narra que tan importante fue el vínculo con su pueblo; la coya influyó en cuanto a la política, pues se encargaba en aconsejar al máximo gobernante, el Inka, y según los expertos la coya portaba un gran conocimiento y gracias a ella el Inka tenía una buena comunicación con el pueblo. En esta investigación, se comparte esa opinión puesto que en las primeras láminas encontradas destaca la primera coya quien fue una excelente gobernante, además era diferenciada por su gran belleza, pero no con todas las coyas era así. Como se observa en la quinta coya, que, por sufrir una enfermedad, ella en su estado se lastimaba, teniendo como resultado el desfigurarse el rostro y arrancarse el cabello. Algunas coyas tenían tantas servidumbres, que tenían sirvientas enanas, esto por la preferencia de algunas coyas, otras ayudaban al pueblo, y tal como se menciona, algunas tenían cierta cercanía con la brujería, según el cronista, porque realizaban milagros a la gente del pueblo.

Cornejo (2019), registró y caracterizó diferentes aspectos culturales de los poquecollas, grupo étnico graficado por Guaman Poma en sus láminas. También Cornejo (2020; 1999), nos brinda una nueva visión de la provincia Inka de Pachacamac, lo que apoya nuestra investigación en el tema Inka.

\section{Conclusión}

Como conclusión general, el análisis iconológico y la cultura material, se relacionan de manera que este último permite identificar el origen y vida de las coyas a través de elementos, en este caso de la vestimenta que portaban, logrando así darles una interpretación a las características principales de cada coya.

En cuanto a la escena y la cultura material, vemos que Guamá Poma de Ayala jugaba con la composición de las ilustraciones en distintos planos, la coya se presenta en escena, en el espacio como personaje principal, ya que siempre se distingue claramente de otros elementos.

La cultura material nos permite categorizar los objetos, y a través de estos lograr identificar la personalidad, preferencias, comportamiento, aspecto físico de los personajes dueños de los objetos, lo cual es la base del estudio de la cultura material, identificar a un individuo por sus posesiones.

Las coyas tenían un papel importante en la política, porque junto al gobernante inka se encargaban de dirigir y tener control sobre el pueblo; en el caso de los objetos materiales o cultura material encontramos el acsu, la lliclla y el tupu, porque representaban poderío y eran símbolo de la mujer noble inka.

En cuanto a la sociedad y la cultura material, las coyas ayudaban al pueblo, incluso estas tenían "sirvientes", lacayos ancianos y enanas, diferenciando clases sociales.

\section{Recomendación}

Se recomienda tener un estudio de investigación que pueda ser de interés o ayude a nuestro país y sociedad. Con el tema de las coyas podrá este trabajo ser de utilidad para estudiantes e investigadores que busquen información sobre este personaje en el Tawantinsuyu. Creemos que brinda nuevos aportes al conocimiento de la historia de los inkas en varios aspectos de la cultura material. Al ser un tema novedoso por su pluralidad disciplinaria, abre caminos nuevos de investigación multidisciplinarias.

También se sugiere, poder incorporar estos temas multidisciplinarios en la elaboración de tesis de grado y posgrado y motivar futuras investigaciones en las disciplinas de historia, historia del arte, arqueología y diseño gráfico.

\section{Bibliografía}

Alvez, R. (2016). La mujer Inka según el cronista Guaman Poma de Ayala (siglos XVI al XVII). Recuperado de http://cerpdellitoral.cfe.edu.uy/index.php/academico/ departamentos

Baulenas, A. (2015). Señora de todas las tierras: Mama Ocllo y el papel de la Coya en el Tahuantinsuyo. Recuperado de: http://www.revistaandinacbc.com/wpcontent/uploads/2016/ra53/ra-53-2015-11.PDF

Cornejo, M. (2020). Inka Province of Pachacamac, Archaeological Analysis of an Inka Province of Pachacamac and the Ischma nation on the Central Coast of Peru. LAP LANDER Academic Publishing. 
KS OmniScriptum Publishing Brivibas gatve 197 LV1039 Riga, Latvia.

Cornejo, M. (1999). An Archaeological Analysis of an Inka Province: Pachacamac and the Ischma nation of the central coast of Perú. Tesis para obtener el grado de Doctor en Arqueología, (Ph.D). Department of Archaeology and Anthropology, Australian National University. Canberra, Australia. Tesis presentada el 28 de mayo de 1999 y aprobada en mayo del 2000.

Cornejo, M. (2019). Red Vial Puquina, migraciones y el Qhapaq Nam. En: Arqueología Peruana del COARPE (ArqPerCOARPE). Revista del Colegio Profesional de Arqueólogos del Perú / ISSN2664-147X, No. 2, 2019: 13-27, Lima, Perú.

Dias, J. (2016). Muerte y escatología: Transcendencia medieval a través de la imagen. Un análisis historiográfico, iconográfico e iconológico sobre la muerte en la sociedad medieval y en la tradición pictórica del norte de Europa en los siglos XV y XVI (tesis de doctorado). Recuperado de http://digibug.ugr.es/ handle/10481/43407

Gaston, R. (1998). Review: Erwin Panofsky and the Classical Tradition. Recuperado https://www.jstor.org/ stable/30222412?seq=1

Guaman P., F. (1993 [1615]). Nueva Coronica y Buen Gobierno I. Perú: FCE.
Guaman P., F. (1993). Nueva coronica y buen gobierno I. Recuperado de http://www.latinamericanstudies.org/ Inkas/Nueva_coronica_1.pdf

Palomino, P. (2018). Análisis iconológico de las coyas a través de la cultura material de Guaman Poma de Ayala, Lima, 2019. Tesis para optar el grado de licenciada en la EAP de ADGE de la Facultad de CC. CC. de la UCV, Lima, 2019.

Panofsky, E. (1955). El significado de las artes visuales. Recuperado de: http://soda.ustadistancia.edu.co/enlinea/artes_creacion-texto-y-cont-esteticahistarte_ricardosuarezI/Significado_artes_visuales_Erw[1].pdf

SAAvedra, M. (2017). La mujer del gobernante: la Coya: Recuperado de https://www.artehistoria.com/es/ contexto/la-mujer-del-gobernante-la-coya

SÁNCHEZ, J. (2014). Iconología simbólica en los bordados populares toledanos (tesis de doctorado). Recuperado de https://eprints.ucm.es/25010/1/T35257.pdf

UlLOA, J. (2015). Cultura material, patrimonio arqueológico y diáspora africana en la República Dominicana. un enfoque crítico-epistemológico (tesis de licenciatura). Recuperado de http://eds.a.ebscohost.com/eds/pdfviewer/pdfviewer?vid=3 \&sid=15b87fac-c77b-493e8024-50e506f67050\%40sdc-v-sessmgr03 